\title{
TRANSFORM SOLUTIONS OF DIRICHLET PROBLEMS IN QUARTER-SPACES
}

\author{
by I. N. SNEDDON
}

(Received 7 September, 1973)

1. In a recent paper, Boudjelkha and Diaz [1] have considered the solutions of the Dirichlet problems for Laplace's equation

and for Helmholtz's equation

$$
\Delta_{n} w=0
$$

$$
\left(\Delta_{n}-\lambda^{2}\right) w=0 \quad(\lambda \in \mathbb{R})
$$

in $\frac{1}{4}-\mathbb{R}^{n}$. The purpose of this brief note is to show that their formulae may be derived easily by the use of the theory of multiple Fourier transforms.

2. We shall use the notation $\mathrm{z}=\left(z_{1}, z_{2}, \ldots, z_{n-2}\right)$ for the position vector of a point in $\mathbb{R}^{n-2}$ and $(x, y, \mathbf{z})=\left(x, y, z_{1}, z_{2}, \ldots, z_{n-2}\right)$ for that of a point in $\mathbb{R}^{n}$.

We consider the solution of the Dirichlet problem

$$
\begin{aligned}
\Delta_{n} w(x, y, \mathrm{z}) & =0, \quad x>0, \quad y>0, \quad \mathrm{z} \in \mathbb{R}^{n-2} \\
w(x, 0, \mathrm{z}) & =f(x, \mathrm{z}), \quad x>0, \quad \mathrm{z} \in \mathbb{R}^{n-2} \\
w(0, y, \mathrm{z}) & =g(y, \mathrm{z}), \quad y>0, \quad \mathrm{z} \in \mathbb{R}^{n-2} \\
w(x, y, \mathrm{z}) & \rightarrow 0 \text { as } \quad r \rightarrow \infty \quad \text { with } \quad x>0, y>0,
\end{aligned}
$$

where the functions $f$ and $g$ are prescribed and $f(0, \mathbf{z})=g(0, \mathbf{z})$ for all $\mathbf{z} \in \mathbb{R}^{n-2} ; r$ is the radial distance defined by $r^{2}=x^{2}+y^{2}+|z|^{2}$.

We also consider the solution of the Dirichlet problem

$$
\begin{array}{rlrl}
\left(\Delta_{n}-\lambda^{2}\right) w(x, y, \mathbf{z}) & =0 & & \left(x>0, y>0, \mathbf{z} \in \mathbb{R}^{n-2}\right), \\
w(x, 0, \mathbf{z}) & =f(x, \mathbf{z}) & & \left(x>0, \mathbf{z} \in \mathbb{R}^{n-2}\right), \\
w(0, y, \mathbf{z}) & =g(y, \mathbf{z}) & & \left(y>0, \mathbf{z} \in \mathbb{R}^{n-2}\right), \\
w(x, y, \mathbf{z}) & \rightarrow 0 \text { as } \quad r \rightarrow \infty \text { with } x>0, y>0 .
\end{array}
$$

To solve the equations (2.1) and (2.5) we introduce two transforms $\mathscr{S}_{(n-1)}$ and $\mathscr{C}_{(n-1)}$. Employing the usual notation

$$
\mathscr{F}_{(n-2)}[f(x, y, \mathbf{z}) ; \mathbf{z} \rightarrow \alpha]=(2 \pi)^{-\frac{1}{2 n-1}} \int_{\mathbb{R}^{n-2}} f(x, y, \mathbf{z}) \exp \{i(\alpha \cdot \mathbf{z})\} d \mathbf{z},
$$

with $\alpha=\left(\alpha_{1}, \alpha_{2}, \ldots, \alpha_{n-2}\right),(\alpha . z)=\left(\alpha_{1} z_{1}+\ldots+\alpha_{n-2} z_{n-2}\right)$, and

$$
\mathscr{F}_{s}[f(x, y, \mathbf{z}) ; x \rightarrow \xi]=(2 / \pi)^{\frac{1}{2}} \int_{0}^{\infty} f(x, y, \mathbf{z}) \sin (\xi x) d x,
$$




$$
\mathscr{F}_{c}[f(x, y, \mathbf{z}) ; x \rightarrow \xi]=(2 / \pi)^{\frac{1}{2}} \int_{0}^{\infty} f(x, y, \mathbf{z}) \cos (\xi x) d x
$$

for the operators of the multiple Fourier transform and the sine and cosine transform, we define $\mathscr{S}_{(n-1)}$ and $\mathscr{C}_{(n-1)}$ by the equations

$$
\hat{\chi}_{(n-1)}^{s}(\xi, y, \alpha) \equiv \mathscr{S}_{(n-1)}[\chi(x, y, \mathbf{z}) ; x \rightarrow \xi, \mathbf{z} \rightarrow \alpha]=\mathscr{F}_{(n-2)}\left[\mathscr{F}_{s}\{\chi(x, y, \mathbf{z}) ; x \rightarrow \xi\} ; \mathbf{z} \rightarrow \alpha\right]
$$

and

$$
\hat{\chi}_{(n-1)}^{c}(\xi, y, \alpha) \equiv \mathscr{C}_{(n-1)}[\chi(x, y, z) ; x \rightarrow \xi, z \rightarrow \alpha]=\mathscr{F}_{(n-2)}\left[\mathscr{F}_{c}\{\chi(x, y, z) ; x \rightarrow \xi\} ; \mathbf{z} \rightarrow \alpha\right]
$$

respectively. If we denote the inverse of $\mathscr{F}_{(n-2)}$ by $\mathscr{F}_{(n-2)}^{*}$, so that

$$
\mathscr{F}_{(n-2)}^{*}[F(\alpha) ; \alpha \rightarrow \mathrm{z}]=\mathscr{F}_{(n-2)}[F(\alpha) ; \alpha \rightarrow-\mathrm{z}],
$$

we can easily show that the inverses $\mathscr{S}_{(n-1)}^{*}, \mathscr{C}_{(n-1)}^{*}$ of $\mathscr{S}_{(n-1)}, \mathscr{C}_{(n-1)}$ are defined by the formulae

$$
\begin{aligned}
& \chi(x, y, \mathbf{z}) \equiv \mathscr{S}_{(n-1)}^{*}\left[\hat{\chi}_{(n-1)}^{s}(\xi, y, \alpha) ; \xi \rightarrow x, \alpha \rightarrow \mathrm{z}\right]=\mathscr{F}_{(n-2)}^{*}\left[\mathscr{F}_{s}\left\{\hat{\chi}_{(n-1)}^{s}(\xi, y, \alpha) ; \xi \rightarrow x\right\} ; \alpha \rightarrow \mathrm{z}\right] \\
& \chi(x, y, \mathbf{z}) \equiv \mathscr{C}_{(n-1)}^{*}\left[\hat{\chi}_{(n-1)}^{c}(\xi, y, \alpha) ; \xi \rightarrow x, \alpha \rightarrow \mathrm{z}\right]=\mathscr{F}_{(n-2)}^{*}\left[\mathscr{F}_{c}\left\{\hat{\chi}_{(n-1)}^{c}(\xi, y, \alpha) ; \xi \rightarrow x\right\} ; \alpha \rightarrow \mathrm{z}\right]
\end{aligned}
$$

Using the convolution theorem for multiple Fourier transforms and the result

$$
2 \int_{0}^{\infty} F_{s}(\xi) \phi_{c}(\xi) \sin (\xi x) d \xi=\int_{0}^{\infty} f(t)\{\phi(|t-x|)-\phi(t+x)\} d t,
$$

where $F_{s}=\mathscr{F}_{s} f, \phi_{c}=\mathscr{F}_{c} \phi$ (cf. [3, p. 63]), we deduce that

$$
\begin{aligned}
& \mathscr{S}_{(n-1)}^{*}\left[f_{(n-1)}^{s}(\xi, \alpha) \hat{\phi}_{(n-1)}^{c}(\xi, y, \alpha) ; \xi \rightarrow x, \alpha \rightarrow \mathbf{z}\right] \\
& \quad=2^{-\frac{1}{2}(n-3)} \pi^{-\frac{1}{2}(n-1)} \int_{0}^{\infty} d s \int_{\mathbb{R}^{n-2}} f(s, \mathbf{t})\{\phi(|x-s|, y, \mathbf{z}-\mathbf{t})-\phi(x+s, y, \mathbf{z}-\mathbf{t})\} d \mathbf{t} .
\end{aligned}
$$

3. If we apply the operator $\mathscr{S}_{(n-1)}$ to both sides of equation (2.1) and assume that $w(0, y, z) \equiv 0$, we find that the equation is transformed to

which has a solution

$$
\left(\frac{\partial^{2}}{\partial y^{2}}-\xi^{2}-|\alpha|^{2}\right) \hat{w}_{(n-1)}^{s}(\xi, y, \alpha)=0
$$

where

$$
\hat{w}_{(n-1)}^{s}(\xi, y, \alpha)=A(\xi, \alpha) \hat{\phi}_{(n-1)}^{c}(\xi, y, \alpha),
$$

$$
\hat{\phi}_{(n-1)}^{c}(\xi, y, \alpha)=\exp \left\{-y \sqrt{ }\left(\xi^{2}+|\alpha|^{2}\right)\right\}
$$

Inverting (3.1), we see that equation (2.1) has solutions of the form

$$
w(x, y, z)=\mathscr{S}_{(n-1)}^{*}\left[A(\xi, \alpha) \hat{\phi}_{(n+1)}^{c}(\xi, y, \alpha) ; \xi \rightarrow x, \alpha \rightarrow z\right] .
$$

Also, if we write

$$
\phi_{(n-1)}(x, y, z)=\mathscr{C}_{(n-1)}^{*}\left[\hat{\phi}_{(n+1)}^{c}(\xi, y, \alpha) ; \xi \rightarrow x, \alpha \rightarrow z\right]
$$


and make use of a well-known result from the theory of integral transforms $([3, \mathrm{p} .82])$, we see that

$$
\phi_{(n-1)}(x, y, \mathbf{z})=\mathscr{F}_{(n-1)}\left[\hat{\phi}_{(n-1)}^{c}(\xi, y, \alpha) ; \xi \rightarrow x, \alpha \rightarrow \mathbf{z}\right]=r^{-v} \mathscr{H}_{v}\left[t^{v} e^{-t y} ; r\right],
$$

where $\mathscr{H}_{v}$ is the Hankel transform of order $v=\frac{1}{2}(n-3)$ defined by

$$
\mathscr{H}_{v}[f(t) ; r]=\int_{0}^{\infty} t f(t) \mathrm{J}_{v}(r t) d t .
$$

From formula (8) on p. 182, Vol. I of [2], we deduce immediately that

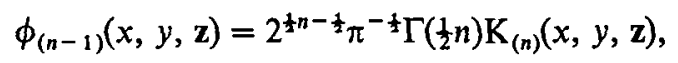

where

$$
\mathbf{K}_{(n)}(x, y, \mathbf{z})=y\left(x^{2}+y^{2}+|\mathbf{z}|^{2}\right)^{-\frac{1}{2} n} .
$$

It follows from equation (3.3) that

$$
\begin{aligned}
w(x, y, z)=\mathscr{S}_{(n-1)}^{*}\left[A(\xi, \alpha) \hat{\phi}_{(n-1)}^{c}(\xi, y, \alpha) ; \xi\right. & \rightarrow x, \alpha \rightarrow \mathrm{z}] \\
& +\mathscr{S}_{(n-1)}^{*}\left[B(\zeta, \alpha) \hat{\phi}_{(n-1)}(\zeta, x, \alpha) ; \zeta \rightarrow y, \alpha \rightarrow \mathrm{z}\right]
\end{aligned}
$$

is a solution of equation (2.1). Since

$$
w(x, 0, \mathbf{z})=\mathscr{S}_{(n-1)}^{*}[A(\xi, \boldsymbol{\alpha}) ;(x, \mathbf{z})], w(0, y, \mathbf{z})=\mathscr{S}_{(n-1)}^{*}[B(\zeta, \boldsymbol{\alpha}) ;(y, \mathbf{z})],
$$

it follows that it will be a solution of the first Dirichlet problem if

$$
\begin{aligned}
& A(\xi, \alpha)=\hat{f}_{(n-1)}^{s}(\xi, \alpha) \equiv \mathscr{S}_{(n-1)}[f(x, \mathrm{z}) ; x \rightarrow \xi, \mathrm{z} \rightarrow \alpha] \\
& B(\zeta, \alpha)=\hat{g}_{(n-1)}^{s}(\xi, \alpha) \equiv \mathscr{S}_{(n-1)}[g(y, \mathrm{z}) ; y \rightarrow \zeta, \mathrm{z} \rightarrow \alpha] .
\end{aligned}
$$

In this way we obtain the solution

$$
\begin{aligned}
w(x, y, z)= & \mathscr{S}_{(n-1)}^{*}\left[\hat{f}_{(n-1)}^{s}(\xi, \alpha) \phi_{(n-1)}^{c}(\xi, y, \alpha) ; \xi \rightarrow x, \alpha \rightarrow \mathrm{z}\right] \\
& +\mathscr{S}_{(n-1)}^{*}\left[\hat{g}_{(n-1)}^{s}(\zeta, \alpha) \hat{\phi}_{(n-1)}^{c}(\zeta, x, \alpha) ; \zeta \rightarrow y, \alpha \rightarrow \mathrm{z}\right] .
\end{aligned}
$$

From equations (2.10), (3.4) and (3.5) we deduce that

$$
\begin{aligned}
w(x, y, \mathbf{z})= & \gamma_{n} \int_{0}^{\infty} d s \int_{\mathbb{R}^{n-2}} f(s, \mathbf{t})\left\{\mathbf{K}_{(n)}(|x-s|, y, \mathbf{z}-\mathbf{t})-\mathbf{K}_{(n)}(x+s, y, \mathbf{z}-\mathbf{t})\right\} d \mathbf{t} \\
& +\gamma_{n} \int_{0}^{\infty} d s \int_{\mathbb{R}^{n-1}} g(s, \mathbf{t})\left\{\mathbf{K}_{(n)}(x,|y-s|, \mathbf{z}-\mathbf{t})-\mathbf{K}_{(n)}(x, y+s, \mathbf{z}-\mathbf{t})\right\} d \mathbf{t},(3.6)
\end{aligned}
$$

where $\mathrm{K}_{(n)}(x, y, z)$ is defined by equation (3.5) and the constant $\gamma_{n}$ by the equation

$$
\gamma_{n}=2 \pi^{-\frac{1}{2} n} \Gamma\left(\frac{1}{2} n\right) \text {. }
$$

4. Similarly, if we apply the operator $\mathscr{S}_{(n-1)}$ to both sides of equation (2.5) and make use of the boundary conditions (2.6) and (2.7), we obtain the solution 
where

$$
\begin{aligned}
v(x, y, \mathbf{z})= & \mathscr{S}_{(n-1)}^{*}\left[\hat{f}_{(n-1)}^{s}(\xi, \alpha) \hat{\psi}_{(n-1)}^{c}(\xi, y, \alpha) ; \xi \rightarrow x, \alpha \rightarrow \mathbf{z}\right] \\
& +\mathscr{S}_{(n-1)}^{*}\left[\hat{g}_{(n-1)}^{s}(\zeta, \alpha) \hat{\psi}_{(n-1)}^{c}(\zeta, x, \alpha) ; \zeta \rightarrow y, \alpha \rightarrow \mathbf{z}\right]
\end{aligned}
$$

Hence

$$
\hat{\psi}_{(n-1)}^{c}(\xi, y, \alpha ; \lambda)=\exp \left\{-y \sqrt{ }\left(\xi^{2}+|\alpha|^{2}+\lambda^{2}\right) .\right.
$$

$$
\psi_{(n-1)}(x, y, z) \equiv \mathscr{C}_{(n-1)}^{*}\left[\hat{\psi}_{(n-1)}^{c}(\xi, y, \alpha) ;(x, \mathrm{z})\right]=r^{-v} \mathscr{H}_{v}\left[t^{v} \exp \left\{-y \sqrt{ }\left(t^{2}+\lambda^{2}\right)\right\} ; r\right]
$$

again with $v=\frac{1}{2}(n-3)$. Making use of formula (19) on p. 31, Vol. II of [2], we find that

where

$$
\psi_{(n-1)}(x, y, \mathbf{z})=2^{\frac{1}{2}} \pi^{-\frac{1}{2}} \lambda^{\frac{1}{2} n} \mathrm{~L}_{(n)}(x, y, \mathbf{z} ; \lambda),
$$

$$
\mathrm{L}_{(n)}(x, y, \mathrm{z} ; \lambda)=y r^{-n+3 / 4} \mathrm{~K}_{\frac{1}{2} n}(\lambda r), \quad\left(r^{2}=x^{2}+y^{2}+|z|^{2}\right)
$$

and $\mathrm{K}_{m}$ denotes the Bessel function of the second kind of order $m$. Making use of equation (2.10), we have, finally,

$$
\begin{aligned}
v(x, y, \mathbf{z})= & \Gamma_{n} \int_{0}^{\infty} d s \int_{\mathbb{R}^{n-2}}^{\infty} f(s, \mathbf{t})\left\{\mathrm{L}_{(n)}(|x-s|, y, \mathbf{z}-\mathbf{t})-\mathrm{L}_{(n)}(x+s, y, \mathbf{z}-\mathbf{t})\right\} d \mathbf{t} \\
& +\Gamma_{n} \int_{0}^{\infty} d s \int_{\mathbb{R}^{n-2}} g(s, \mathbf{t})\left\{\mathrm{L}_{(n)}(x,|y-s|, \mathbf{z}-\mathbf{t})-\mathrm{L}_{(n)}(x, y+s, \mathbf{z}-\mathbf{t})\right\} d \mathbf{t},
\end{aligned}
$$

where the constant $\Gamma_{n}$ is given by the equation

$$
\Gamma_{n}=2^{\frac{1}{2} n} \pi^{-\frac{1}{2} n} \lambda^{\frac{1}{2} n}
$$

5. There is a simple relation between the kernels $K_{(n+1)}$ and $L_{(n)}$. If we write $\mathrm{z}^{+}=\left(z_{1}, z_{2}, \ldots, z_{n-1}\right)$, then, from equations (3.2) and (4.2), we deduce that

$$
\hat{\phi}_{(n)}^{c}\left(\xi, y, \alpha, \alpha_{n-1}\right)=\hat{\psi}_{(n-1)}^{c}\left(\xi, y, \alpha, \alpha_{n-1}\right) .
$$

Applying the operator $\mathscr{S}_{(n)}^{*}$ to both sides, we find that

i.e. that

$$
\phi_{(n)}\left(x, y, \mathbf{z}, z_{n-1}\right)=\mathscr{F}^{*}\left[\psi_{(n-1)}\left(x, y, \mathbf{z}, \alpha_{n-1}\right) ; \alpha_{n-1} \rightarrow z_{n-1},\right.
$$

and hence that

$$
\mathscr{F}\left[\phi_{(n)}\left(x, y, \mathbf{z}^{+}\right) ; z_{n-1} \rightarrow \lambda\right]=\psi_{(n-1)}(x, y, \mathbf{z} ; \lambda)
$$

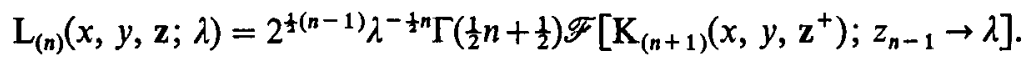

\section{REFERENCES}

1. M. T. Boudjelkha and J. B. Diaz, Applicable analysis 2, 1 (1972).

2. A. Erdèlyi (editor), Tables of integral transforms (New York, 1954).

3. I. N. Sneddon, The use of integral transforms (New York, 1972).

\section{UNIVERSITY OF GLASGOW}

GLASGOW G12 8QW 